\title{
Uso de óleos essenciais para o controle de pragas do milho
}

\section{Ana Paula Mura Nastari Mattos ${ }^{1}$, Analu Monalise Aragão Krewer ${ }^{1}$, Camila Eccel $^{1}$ and Juliano Will ${ }^{1}$}

\section{Resumo}

O Brasil é um dos maiores produtores de milho do mundo. O clima e o surgimento de pragas são fatores que interferem na quantidade e qualidade desta produção agrícola. No que se refere às pragas presentes nas lavouras, o seu combate com a utilização de agrotóxicos demonstrase eficiente, no entanto, se mal-empregados, podem oferecer riscos à saúde humana e ao meio ambiente. Diversos estudos apontam os óleos essenciais como alternativa para substituição ao uso de agrotóxicos. A proposta deste trabalho consistiu em apresentar uma revisão bibliográfica a respeito da eficiência da utilização de óleos essenciais com efeito inseticida e repelente para o combate às diferentes pragas do milho. Os resultados demonstram que o óleo essencial de Baccharis dracunculifolia apresentou efeito inseticida significativo em aplicação contra os insetos; o óleo essencial do alecrim-pimenta (Lippia sidoides) pode ser utilizado como controle alternativo contra o fungo Curvularia; o óleo essencial das folhas de mentrasto (Ageratum conyzoides) apresentou um resultado precoce no controle da lagarta-do-cartucho; o óleo essencial das folhas da aroeira-salso (Schinus molle L) apresentou eficiência no controle do gorgulho-do-milho (Sitophiluz zeamais), assim como o óleo essencial de cravo-de-defunto (Tagetes patula) também apresentou resultado positivo no combate a esta praga. O óleo essencial de folhas de pimentalonga possui atividade inseticida contra a lagarta-do-cartucho com eficiência na mortalidade. Os óleos essenciais do marmeleio (Croton heliotropiifolius), velame (Croton pulegiodorus), aroeira (Myracrodruon urundeuva) e manjericão (Ocimum basilicum) apresentou resultados positivos no combate ao besouro-castanho (Tribolium castaneum); os óleos essenciais de citronela, tomilho, capim-limão, eucalipto, canela e cravo controlam com eficiência a germinação de conídios e o crescimento micelial de Stenocarpella maydis nas sementes do milho. Os óleos essenciais de cravo-da-índia e canela reduziram a incidência de patógenos e os óleos essenciais de cravo-daíndia e tomilho atuaram diretamente no controle do fungo conídio. Ainda, os óleos essenciais de eucaliptos revelaram-se promissores na proteção de plantações quando pulverizados nas mesmas. Ou seja, diversos óleos essenciais apresentaram diferentes aplicabilidades no controle das pragas do milho.

\section{Palavras-chave}

armazenamento de grãos, biodegradável, inseticida natural, manejo integrado de pragas, repelente botânico 


\section{Introdução}

O milho (Zea mays L.) é extremamente utilizado em uma grande diversidade de pratos alimentícios, não somente no Brasil, mas em toda extensão mundial. Além disso, é o principal ingrediente utilizado em formulações para rações de aves, bovinos e suínos, tornando-se o cereal mais plantado no mundo. De acordo com o último censo agropecuário foram produzidas 88 milhões de toneladas de milho em grão no Brasil (Ibge, 2019).

As perdas devido ao ataque de pragas chegam a $20 \%$ da produção total do grão, demandando a necessidade de novas pesquisas e tecnologias (Silva et al., 2007). Atualmente, o método de controle de pragas do milho mais utilizado é através da aplicação de agrotóxicos, mas apesar de sua eficácia, seu uso intensivo pode acarretar o acúmulo de resíduos tóxicos nos alimentos de consumo humano e animal, contaminação do meio ambiente, surgimento de resistência entre os insetos, entre outros (Campos et al., 2014).

Desta forma, pesticidas naturais que possuem efeito inseticida e repelente que não ocasionam danos à população e aos recursos naturais, apresentam-se como alternativa no manejo integrado de pragas (Dietrich, Aparecida, Strohschoen, Schultz, \& Deise, 2011). Dentre os pesticidas naturais, destacam-se os óleos essenciais, também denominados de óleos voláteis, óleos etéreos ou essências (Pereira et al., 2014).

Os óleos essenciais são compostos orgânicos aromáticos voláteis de ocorrência natural extraídos das plantas. O termo "óleo" é devido ao seu aspecto oleoso, pouco miscível em água. Frequentemente apresentam odor marcante, característica das funções inseticida e repelente (Morais \& De, 2009).

As plantas possuem mecanismos naturais contra insetos, os chamados metabólitos secundários, e possuem funções de adaptar o organismo vegetal ao habitat em que vivem. Os metabólitos secundários protegem as plantas contra o ataque de pragas e infecções microbianas, além de apresentar características fitoterápicas (Freitas et al., 2020).

Os metabólitos secundários, presentes nos óleos essenciais são os responsáveis pelos principais odores encontrados nas partes da planta (Fabiane, Ferronatto, Santos, \& Onofre, 2008). Os óleos essenciais têm se mostrado eficazes no controle de pragas de produtos armazenados, além de serem considerados inseticidas ecológicos, que apresentam um menor impacto ambiental comparado aos inseticidas sintéticos, são biodegradáveis e possuem capacidade de serem aplicados em várias culturas (Zimmermann et al., 2020).

O efeito repelente dos óleos essenciais é uma das características mais significativas no controle de pragas de grãos armazenados, visto que a infestação será menor quanto maior for a repelência ao óleo, pelo fato de favorecer a diminuição da postura e o número de eclosões dos insetos (Coitinho, Oliveira, De;, Câmara, \& Da, 2006) (Coitinho et al., 2006).

\footnotetext{
${ }^{11}$ Programa de PósGraduação em Tecnologia e Ambiente, Instituto Federal Catarinense, Araquari, Santa Catarina, Brasil

Corresponding author:

Ana Paula Mura Nastari Mattos, 'Programa de PósGraduação em Tecnologia e Ambiente, Instituto Federal Catarinense, Araquari, Santa Catarina, Brasil

Email: ana.nastari@hotmail.com
} 
A extração dos óleos essenciais pode ser realizada por diversas metodologias, entre elas: a hidrodestilação e a destilação por arraste a vapor. Na hidrodestilação o material vegetal fica submerso na água e é aquecido até o ponto de ebulição para extração dos óleos essenciais. Já na destilação por arraste a vapor, o material vegetal é inserido em destilador e o vapor é pressionado sobre o material de forma que o vapor auxilia a liberação das moléculas aromáticas do material vegetal, onde os óleos voláteis são carregados pelo vapor (Sefidkon, Abbasi, Jamzad, \& Ahmadi, 2007).

Os elementos voláteis dos óleos podem ser concentrados em partes anatômicas específicas, dependendo da família e espécie da planta, como em raízes, madeira, casca do tronco, folha, flor, entre outras partes. Também interferem na composição do óleo essencial as condições externas, como horário da coleta, condições de clima e solo, localização geográfica, ciclo vegetativo da espécie, entre outros (Fabiane et al., 2008).

O modo de ação dos óleos essenciais no sistema nervoso dos insetos está diretamente relacionado com a rapidez na mortalidade. Há evidências sobre a interferência dos óleos no neuromodulador octopamina, que é encontrado em todos os invertebrados (Corrêa \& Salgado, 2011). A octopamina é semelhante à noradrenalina e age como neurohormônio, neuromodulador e neurotransmissor, regulando os batimentos cardíacos, os movimentos, o comportamento e o metabolismo dos insetos (Roeder, 1999).

Sendo assim, em função destas características, a proposta deste artigo consiste em realizar um levantamento bibliográfico sobre a eficácia da utilização de óleos essenciais com efeito inseticida e repelente no combate às diferentes pragas do milho.

\section{Metodologia de Trabalho}

Realizou-se uma busca computadorizada por artigos de relevância que tratam de estudos sobre óleos essenciais como inseticidas agrícolas, utilizando as bases de dados MedLine, Portal da Capes, Science Direct e Google Acadêmico. Como limite de tempo, o período compreendido entre 2000 a 2021, e com os termos de busca "óleos essenciais como inseticidas" e "óleos essenciais para o controle de pragas do milho", resultando em 6.050 artigos. Diversos artigos foram selecionados, priorizando publicações de pesquisadores e instituições reconhecidas, com a intenção de explorar e conhecer o que está sendo desenvolvido em relação ao tema deste artigo. Adicionalmente, selecionou-se por busca ativa outros artigos e capítulos de livros que eram pertinentes ao tema a partir das referências bibliográficas presentes nas pesquisas encontradas.

\section{Resultados e discussão}

No levantamento bibliográfico realizado foi encontrado um total de 16 óleos essenciais aplicados em 5 diferentes pragas que acometem o milho.

Tabela 1: Atividade inseticida de óleos essenciais descritos na literatura para a cultura do milho.

Jr et al. (2013) avaliaram o efeito repelente e inseticida do óleo essencial de Baccharis dracunculifolia no controle de Sitophilus zeamais em grãos de milho armazenados e observaram que o óleo essencial de B. dracunculifolia manifestou efeito inseticida significativo somente na dose mais alta de $100 \mu \mathrm{L}$ eapós 48 horas de exposição. No teste de repelência todas as doses do óleo apresentaram efeito repelente sobre os insetos.

A ação antimicrobiana do óleo essencial de alecrim-pimenta (Lippia sidoides) sobre o patógeno Curvularia sp. foi avaliada por (Ferreira et al., 2014). Os resultados obtidos demonstraram que o óleo 
Table 1. Atividade inseticida de óleos essenciais descritos naliteratura para a cultura do milho.

\begin{tabular}{|c|c|c|}
\hline Óleo essencial & Praga & Referência Bibliográfica \\
\hline $\begin{array}{l}\text { Alecrim-do-campo (Baccharis } \\
\text { dracunculifolia) }\end{array}$ & $\begin{array}{l}\text { Gorgulho-do-milho (Sitophilus } \\
\text { zeamais) }\end{array}$ & (Jr et al., 2013) \\
\hline Alecrim Pimenta (Lippia sidoides) & Curvulária (Curvularia sp.) & (Ferreira et al., 2014) \\
\hline $\begin{array}{l}\text { Erva-de-São-João (Ageratum } \\
\text { Conyzoides) }\end{array}$ & $\begin{array}{l}\text { Lagarta-do-cartucho } \\
\text { (Spodoptera frugiperda) }\end{array}$ & (Lima et al., 2010) \\
\hline Aroeira-salso (Schinus molle) & $\begin{array}{l}\text { Gorgulho-do-milho (Sitophilus } \\
\text { zeamais) }\end{array}$ & (Fernandes \& Favero, 2014) \\
\hline $\begin{array}{l}\text { Pimenta Longa (Piper } \\
\text { hispidinervum) }\end{array}$ & $\begin{array}{l}\text { Lagarta-do-cartucho } \\
\text { (Spodoptera frugiperda) }\end{array}$ & (Lima et al., 2009) \\
\hline $\begin{array}{l}\text { Marmeleiro (Croton } \\
\text { heliotropiifolius) }\end{array}$ & $\begin{array}{l}\text { Besouro castanho (Tribolium } \\
\text { castaneum) }\end{array}$ & (Magalhães et al., 2015) \\
\hline Velame (Croton pulegiodorus) & $\begin{array}{l}\text { Besouro castanho (Tribolium } \\
\text { castaneum) }\end{array}$ & Magalhães et al. (2015) \\
\hline $\begin{array}{l}\text { Aroeira (Myracrodruon } \\
\text { urundeuva) }\end{array}$ & $\begin{array}{l}\text { Besouro castanho (Tribolium } \\
\text { castaneum) }\end{array}$ & (Magalhães et al., 2015) \\
\hline Manjericão (Ocimum basilicum) & $\begin{array}{l}\text { Besouro castanho (Tribolium } \\
\text { castaneum) }\end{array}$ & (Magalhães et al., 2015) \\
\hline Cravo-de-defunto (Tagetes patula) & $\begin{array}{l}\text { Gorgulho-do-milho (Sitophilus } \\
\text { zeamais) }\end{array}$ & (Restello et al., 2009) \\
\hline Eucalipto (Eucalyptus sp.) & $\begin{array}{l}\text { Lagarta-do-cartucho } \\
\text { (Spodoptera frugiperda) }\end{array}$ & (Souza et al., 2010) \\
\hline Citronela (Citronella sp.) & $\begin{array}{l}\text { Podridão do Colmo } \\
\text { (Stenocarpella maydis) }\end{array}$ & (Teixeira et al., 2010) \\
\hline Tomilho (Thymus vulgaris) & $\begin{array}{l}\text { Podridão do Colmo } \\
\text { (Stenocarpella maydis) }\end{array}$ & (Teixeira et al., 2010) \\
\hline Capim-limão (Cymbopogon sp.) & $\begin{array}{l}\text { Podridão do Colmo } \\
\text { (Stenocarpella maydis) }\end{array}$ & (Teixeira et al., 2010) \\
\hline Eucalipto (Eucalyptus sp.) & $\begin{array}{l}\text { Podridão do Colmo } \\
\text { (Stenocarpella maydis) }\end{array}$ & (Teixeira et al., 2010) \\
\hline Canela (Cinnamomum sp.) & Podridão do Colmo & (Teixeira et al., 2010) \\
\hline
\end{tabular}


essencial de alecrim pimenta pode ser utilizado como controle alternativo do fungo Curvularia sp. na concentração de $7,5 \mu \mathrm{L} / \mathrm{mL}$, onde não se percebe fitotoxicidade para as plantas de milho.

O trabalho de (Lima et al., 2010) teve como objetivo identificar e quantificar o óleo essencial de folhas de mentrasto (Ageratum conyzoides) e avaliar sua toxicidade para a lagarta-do-cartucho do milho (Spodoptera frugiperda). Observou-se que o precoceno foi o constituinte encontrado em maior quantidade, sendo responsável por $87 \%$ da composição do óleo essencial de A. conyzoides e o efeito inseticida foi constatado para a lagarta-do-cartucho (S. frugiperda).

Fernandes e Favero (2014) testaram a toxicidade de contato, fumigação, repelência e ação tópica do óleo essencial das folhas de Aroeira-salso (Schinus molle) sobre o gorgulho-do-milho (Sitophilus zeamais), uma das principais pragas do milho armazenado no Brasil. O óleo se mostrou eficaz no controle de S. zeamais dependendo da concentração por meio de três vias de intoxicação: contato, fumigação e repelência, apresentando assim efeito insetistático por meio destas vias. $\mathrm{O}$ efeito inseticida foi baixo e observado unicamente por meio da via de intoxicação por aplicação tópica.

Segundo a pesquisa de (Lima et al., 2010), que teve como objetivos a obtenção e caracterização do óleo essencial de folhas de pimenta-longa (Piper hispidinervum) e avaliação de seu efeito no comportamento e/ou mortalidade da lagarta-do-cartucho-do-milho (Spodoptera frugiperda), os resultados demonstraram que o óleo essencial de pimenta-longa possui atividade inseticida sobre S. frugiperda, causando redução alimentar e mortalidade, sendo o safrol (82\%) o composto observado em maior quantidade.

Magalhães et al. (2015) pesquisaram o potencial inseticida dos óleos essenciais de Marmeleiro (Croton heliotropiifolius), Velame (Croton pulegiodorus), Aroeira (Myracrodruon urundeuva) e Manjericão (Ocimum basilicum) sobre adultos de Besouro-castanho (Tribolium castaneum) em milho armazenado. Nos bioensaios de fumigação verificou-se atividade inseticida do óleo essencial de M. urundeuva sobre adultos de T. castaneum. Nos bioensaios de repelência, todos os óleos testados apresentam efeitos repelente. A emergência de T. castaneum reduziu entre 33 e 100\% quando foram criados em pó de milho tratado com os óleos essenciais. Os óleos essenciais de C. pulegiodorus e $\mathrm{O}$. basilicum provocaram atenuação do crescimento populacional de T. castaneum em grãos de milho tratados. Os óleos testados revelaram ser uma alternativa eficiente de controle para o emprego nos programas de manejo de $\mathrm{T}$. castaneum em unidades armazenadoras.

Restello et al. (2009) observaram o efeito repelente e inseticida do óleo essencial de Cravo-dedefunto (Tagetes patula) sobre gorgulho-do-milho (Sitophilus zeamais), na concentração de $10 \mu \mathrm{L}$, sendo, portanto, eficaz no controle de adultos de S. zeamais.

Teixeira, 2010 investigou os efeitos dos óleos essenciais de citronela, tomilho, capim-limão, eucalipto, canela e cravo na germinação de conídios e no crescimento micelial de Stenocarpella maydis e seus efeitos sobre a incidência de S. maydis em sementes de milho inoculadas artificialmente e sobre a germinação e vigor. Os óleos essenciais de cravo-da-índia, canela e tomilho impediram a germinação de conídios em todas as concentrações testadas. Os óleos de cravo-da-índia e canela propiciaram a germinação das sementes em $89,0 \%$ e $84,5 \%$ respectivamente, ao passo que a testemunha não inoculada germinou $93,0 \%$ e a testemunha inoculada $76,5 \%$. Os óleos de cravo-da-índia e canela atenuaram a incidência do patógeno nas sementes para $39,0 \%$ e $28,0 \%$, respectivamente, ao passo que, na testemunha inoculada, a incidência foi de 57,0\%. Os óleos essenciais de cravo-da-índia e tomilho atuaram diretamente sobre o conídio do fungo. 
Souza et al. (2010) analisaram a ação tóxica e fagoinibidora de óleos essenciais de espécies de eucalipto, Corymbia citriodora, Eucalyptus urograndis e E. urophylla para o controle da lagarta-docartucho Spodoptera frugiperda no milho. Os resultados demonstraram que os óleos essenciais testados expressaram toxicidade tópica, sendo o de C. citriodora mais eficiente por causar maior taxa de mortalidade. Os três óleos essenciais evidenciaram atividade antialimentar em relação à testemunha. Os óleos essenciais de C. citriodora e E. urograndis revelaram-se promissores para a proteção do cultivo quando aplicados por pulverização.

Os resultados obtidos demonstram a possibilidade de utilização dos óleos essenciais para o controle das pragas do milho, porém é necessário que sejam realizados outros estudos como, por exemplo, carência, resíduo, custos, alterações organolépticas, entre outros, para que o produtor possa utilizar estes óleos de maneira segura como alternativa ao seu controle.

\section{Conclusão}

Esta pesquisa de referências bibliográficas sobre o uso de óleos essenciais no controle das pragas do milho constata que diversos óleos essenciais apresentam comprovação positiva nas funções inseticida e repelente, tanto no uso individual, como no uso conjunto com outros componentes ativos.

Destacam-se bons resultados para os óleos essenciais de Baccharis dracunculifolia, Lippia sidoides, Ageratum conyzoides, Schinus molle, Piper hispidinervum, Croton heliotropiifolius, Croton

pulegiodorus, Myracrodruon urumbeva, Ocimum basilicum, Tagetes pátula, Eucalyptus sp., Citronella sp., Thymus vulgaris, Cymbopogon sp., Cinnamomum sp. e Syzygium sp.

Com as informações coletadas é possível verificar ação inibidora dos óleos essenciais em soluções preparadas para o combate de fungos e pragas e os testes realizados em plantas de milho delimitaram a concentração máxima a ser utilizada dos compostos majoritários que pode trazer resultados mais conclusivos sobre o real potencial desses óleos para controle.

Sendo assim, constatou-se que diversos óleos essenciais são eficientes no controle das pragas do milho, com o benefício de serem produtos sustentáveis.

\section{Agradecimentos}

Ao professor Adalberto Manoel da Silva pela orientação deste trabalho

\section{References}

Campos, A. C. T., Radunz, L. L., Radünz, A. L., Mossi, A. J., Dionello, R. G., \& Ecker, S. L. (2014). Atividade repelente e inseticida do óleo essencial de carqueja doce sobre o caruncho do feijão. Revista Brasileira de Engenharia Agrícola e Ambiental, 18(8), 861-865.

Coitinho, R. L. B. C., Oliveira, J. V., De;, J., Câmara, M. G. C. G., \& Da, C. A. (2006). ATIVIDADE INSETICIDA DE ÓLEOS VEGETAIS SOBRE INSECTICIDE ACTIVITY OF VEGETAL OILS ON Sitophilus zeamais ( COLEOPTERA : CURCULIONIDAE ) IN STORED. Revista Caatinga, 19(2), 176-182.

Corrêa, J. C. R., \& Salgado, H. R. N. (2011). (Vol. 13). Retrieved from https : / / doi . org/ $10.1590 / \mathrm{s} 1516-05722011000400016$ 
Dietrich, F., Aparecida, A., Strohschoen, G., Schultz, G., \& Deise, A. (2011). Utilização De Inseticidas Botânicos Na Agricultura Orgânica De Arroio Do Meio / Rs Use of Botanical Pesticides in Organic Agriculture in Arroio Do Meio. Rs. R. Bras. Agrociência, 17(2-4), 251-255.

Fabiane, K. C., Ferronatto, R., Santos, A. C., \& Onofre, S. B. (2008). Physicochemical characteristics of the essential oils of Baccharis dracunculifolia and Baccharis uncinella D.C. (Asteraceae). Revista Brasileira de Farmacognosia, 18(2), 197-203.

Fernandes, E. T., \& Favero, S. (2014). Óleo essencial de Schinus molle L. para o controle de Sitophilus zeamais Most.1855 (Coleoptera:Curculionidae) em milho. Revista Brasileira De Agroecologia, 9(1), 225-231.

Ferreira, T. P. S., Santos, G. R., Carlos, D. S., Ságio, S. A., Guimarães, L. G., De, L., .. Da (2014). Efeito do óleo essencial de alecrim pimenta no controle de Curvularia. XXX CONGRESSO NACIONAL DE MILHO E SORGO, 1, 3-6.

Freitas, P. R., Araújo, A. C. J. D., Santos, D., Barbosa, C. R., Muniz, D. F., Rocha, J. E., .. Coutinho, H. D. M. (2020). Characterization and antibacterial activity of the essential oil obtained from the leaves of Baccharis coridifolia DC against multiresistant strains. Microbial Pathogenesis, 145.

Ibge. (2019). Censo agropecuário 2017: resultados definitivos. Censo Agropecuário, 8, 1-105.

Jr, F. W. R., Scariot, M. A., Ecker, S. L., Campos, A. C. T., De, Meneguzzo, M., .. Mossi, A. J. (2013). (Vol. 8). Retrieved from http://revistas.abaagroecologia.org.br/index.php/ cad/article/download/14417/9523

Lima, R. K., Cardoso, M., Das, G., Moraes, J. C., Andrade, M. A., Melo, B. A., \& Rodrigues, V. G. (2010). Caracterização química e atividade inseticida do óleo essencial de Ageratum conyzoides 1. sobre a lagarta-do-cartucho do milho Spodoptera frugiperda (smith, 1797) (Lepidoptera: Noctuidae). Bioscience Journal, 26(1), 1-5.

Lima, R. K., Cardoso, M. G., Moraes, J. C., Melo, B. A., Rodrigues, V. G., \& Guimarães, P. L. (2009). Atividade inseticida do óleo essencial de pimenta longa (Piper hispidinervum C. DC.) sobre lagarta-do-cartucho do milho Spodoptera frugiperda (J. E. Smith, 1797) (Lepidoptera: Noctuidae). Acta Amazonica, 39(2), 377-382.

Magalhães, C. R. I., Oliveira, C. R. F., Matos, C. H. C., Brito, S. S. S., Magalhães, T. A., \& Ferraz, M. S. S. (2015). Potencial inseticida de óleos essenciais sobre Tribolium castaneum em milho armazenado. Revista Brasileira de Plantas Medicinais, 17(4), 1150-1158.

Morais, L. A. S., \& De. (2009). (Vol. 27). Retrieved from http: / /www . alice . cnptia . embrapa .br/alice/handle/doc/577686

Pereira, A. I. S., Pereira, A., Da, G. S., Sobrinho, O. P. L., Cantanhede, E., De, K. P., \& Siqueira, L. F. S. (2014). Atividade antimicrobiana no combate as larvas do mosquito Aedes aegypti: Homogeneização dos óleos essenciais do linalol e eugenol. Educacion Quimica, 25(4), 446-449.

Restello, R. M., Menegatt, C., \& Mossi, A. J. (2009). Efeito do óleo essencial de Tagetes patula L. (Asteraceae) sobre Sitophilus zeamais Motschulsky (Coleoptera, Curculionidae). Revista Brasileira de Entomologia, 53(2), 304-307.

Roeder, T. (1999). Octopamine in invertebrates. Progress in Neurobiology, 59(5), 16-23.

Sefidkon, F., Abbasi, K., Jamzad, Z., \& Ahmadi, S. (2007). The effect of distillation methods and stage of plant growth on the essential oil content and composition of Satureja rechingeri Jamzad. Food Chemistry, 100(3), 1054-1058.

Silva, P. H., Trivelin, P. C. O., Guirado, N., Ambrosano, E. J., Mendes, P. C. D., Rossi, F., . . Milho 
(2007).

CONTROLE ALTERNATIVO DE Sitophilus zeamais MOTS., 1855 (COL.: CURCULIONIDAE), 1855, 902-905.

Souza, T. F., Favero, S., Conte, C., \& De, O. (2010). Revista Brasileira de Agroecologia ISSN : 19809735 Bioatividade de óleos essenciais de espécies de eucalipto para o controle de Spodoptera frugiperda. Smith, 1797 ) (Lepidoptera : Noctuidae), 5, 157-164.

Teixeira, G. A., Potencialidade, Tratamento, Sementes, Óleos, No, . . Milho (2010). (Vol. 1). Retrieved from http://repositorio.ufla.br/jspui/handle/1/2628

Zimmermann, R. C., Aragão, C. E., De, C., Araújo, P. J. P., De, Benatto, A., . . Zawadneak, M. A. C. (2020). Insecticide activity and toxicity of essential oils against two stored-product insects. Crop Protection, 144-144. 